\title{
Provision of a dental office through the techniques of surveys, technology roadmaps and bibliometric analysis
}

\author{
Raúl J. Martelo ${ }^{\# 1}$, Piedad M. Martelo ${ }^{* 2}$, Renzo J. Barrios ${ }^{\# 3}$ \\ ${ }^{\#}$ Faculty of Engineering, University of Cartagena, Cartagena, Bolívar, Colombia \\ 11rmartelog1@unicartagena.edu.co \\ ${ }^{3}$ renzo.barrios1407@gmail.com \\ * Faculty of Health Sciences, University of Cartagena, Cartagena, Bolívar, Colombia \\ 2pmartelog@hotmail.com
}

\begin{abstract}
The purpose of this research is to use the survey techniques, technology roadmaps and bibliometric analysis, to make a consensus among several dentists with the purpose of consulting the required dental devices that have the latest technology available for their practice. The investigation was a qualitative non-experimental descriptive type. The population was made up of teachers from the University of Cartagena, from which a sample of 56 was obtained by means of the census criterion. For data collection, the query and the survey were used. The results showed a chronological list of available technologies, obtained through the consultation of a specific device or dental technology. The conclusions indicate that the techniques eliminate the need for suppliers or expert observers of public entities in the field of dental technology devices for office updates.
\end{abstract}

Keyword - Technological Surveillance, Bibliographic Analysis, Dental Devices, Prospective

\section{INTRODUCTION}

Technological change and globalization increase competition among companies, forcing them to use technology and innovation to carry out processes, as well as making business decisions that do not contain them for development, will result in little sustainability and growth of the company [1].

One field of application of technology is dentistry, which has allowed for better diagnoses, early detection of diseases and treatments through the use of technological devices. This results in the obligation to update or change the devices to continue to offer dental services according to established standards and ensure the quality of their processes [2].

Every time, the dental devices are renewed according to the technological advances and standards of the health entities in Colombia. For this purpose, the supervision of the Colombian Dental Federation (FOC) and the providers of technological articles is requested [3]. These technologies can be determined through the use of techniques, which provide a different way to what is traditionally obtained, for it the technological prospective, offers techniques that collaborate to its recognition, for the novel and useful that result [4].

One of the techniques that can be cited for the purpose described is Technology Roadmaps. It was developed at Motorola to improve the alignment between technology and innovation [5], and was popularized at the beginning of the millennium and was adopted by companies, governments and institutions with technological needs [1]. It has been used as a planning of activities and processes for the formulation of business strategies, in order to determine the rise of new technology. This facilitates the development of research and infrastructure to implement that produce long-term benefits [6].

The Technology Roadmaps have been applied in the automobile sector of Santa Catarina in Brazil [7]. In the measurement of technological gaps for small and medium-sized companies in the Colombian sector, in order to see a consensus of more used technologies [8]. And in the evaluation of the energies obtained through hydrogen in the Canadian industry [9].

Another tool is the Bibliometric Analysis, which has been applied in many researches, such as: the Bibliometric Analysis of the academic contributions in China about burn injuries in research [10], bibliometric citation analysis of the Ebola virus in the period from 1987 to 2015 [11] and the search for articles published from 1997 to 2014 on food waste in China [12].

Although these techniques have applicability in many areas, such as in the medical device industry, they do not have a focus on applications related to dental devices [13], because the technology for the realization of dental processes plays an important part in the work of dentists to ensure the quality of their work [14].

Due to what has been described, the objective of this article is to provide a dental office with current technological devices according to current trends. In this process the SoftProsp web platform was used to provide support with the use of prospective techniques Surveys, Bibliometric Analysis and Technology 
Roadmaps, in addition, the participation of professors from the dentistry faculty of the University of Cartagena was included to know the technological devices used in the offices.

\section{Methodology}

The research developed is of a qualitative, non-experimental, descriptive type. Qualitative because it essentially develops descriptive processes and interprets actions, functionally relevant facts and places them in a broad social context [15], not experimental, because the researcher cannot intentionally manipulate the independent variables or the information obtained from the study subjects [16]; and descriptive because it was aimed at detailing in a natural way the characteristics, factors and procedures present for the change of the devices of the dental office.

\section{1) Population and sample}

In the progress of this study was taken as a case of applying to teachers of the faculty of dentistry from the University of Cartagena consisting of 56 teachers, when considering the census criterion which is not necessary to obtain a sample of the population, it should select all subjects, due to the small size of the population.

\section{2) Data collection materials}

For the collection of data, the consultation with dentists was used to know the opinion of experts and the survey to obtain data of the population studied with respect to the common and current dental devices in a clinic.

\section{3) Validation and reliability}

The validation of the questions designed for data collection was carried out by experts in the field of dentistry, with the aim of eliminating ambiguities and irrelevant aspects [17]. Five dentists participated in this process, expressing impartial opinions to recommend the questions and obtain the necessary concrete information.

\section{4) Data analysis}

The information was organized in alphabetical order and the most common devices among the experts were quantified in a list to classify the five most used in a dental office, for updating them.

\section{5) Research procedure}

For the development of the research, the steps used to update the devices were previously analyzed, based on that, a model was established where the traditional way of updating devices of dental offices and laboratories is determined as shown in Figure 1.

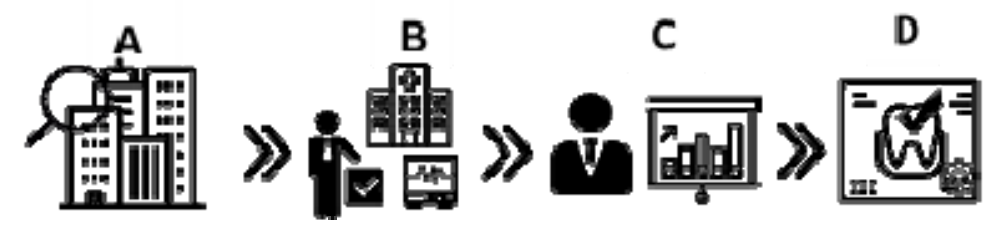

Fig. 1: Traditional scheme for updating the devices of a dental laboratory

The traditional processes for updating laboratories are carried out in the following phases:

1) Self-assessment of infrastructure conditions: health companies must comply with the Mandatory Healthcare Quality Assurance System [3], which establishes technological and scientific capacity conditions that entities must comply with. The company self-evaluates the infrastructure to verify that it has the minimum and indispensable devices for its operation.

2) Verification of the technological infrastructure through a public observer or provider: After verifying that the health entity has what it takes to function, every two years of operation, a consultant from a supplier is contracted or request from the Colombian Federation of Dentists an observer to verify the technological and scientific conditions in which the entity is located.

3) Result of the evaluation of the provider or public observer: At the end of the review of the technological and scientific infrastructure, a list of electronic devices and equipment that require replacement is made. To obtain it, the existence in the Colombian market is checked, creating a list with the quotation so that the entity has selection options.

4) Updating of devices: The health entity through a provider in the city can request new technological devices, the entity delivers the list created by the observer or representative of the supplier to carry out the orders and acquire it according to the stipulated time.

In this procedure you have the possibility of hiring a representative of the provider for the verification of the devices, in order to improve the quality of the services provided to the users, ruled by the Mandatory System of Quality Assurance of the Care of the Health. 
For the improvement of the process of selection of elements of a dental laboratory, a model is proposed in which the use of the techniques Survey, Bibliometric Analysis and Technology Roadmap are added, with the support of the SoftProsp web platform, designed to facilitate the realization of prospective studies from any computer with internet access [18]. The proposed model is shown in Figure 2:

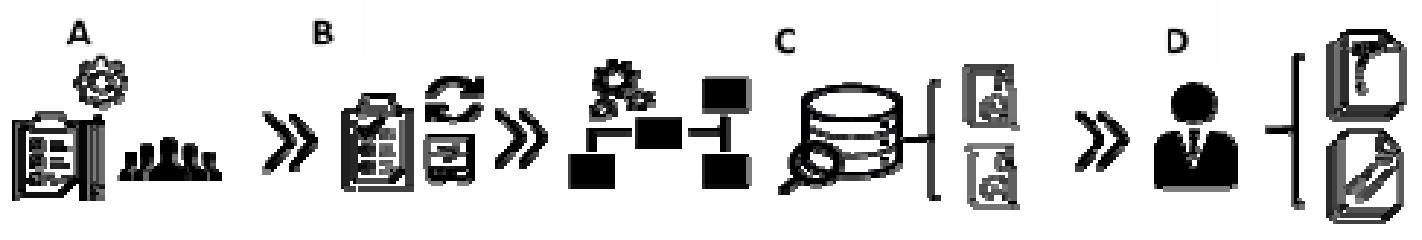

Fig. 2: Proposed model for updating a laboratory.

The model consists of the following phases:

1) Preparation and conduct of surveys of expert population: In this phase the questions are elaborated with the help of the expert dentists, which are part of the survey available in the SoftProsp platform, to apply it to the participants. The purpose is to look for the most used technological devices with the latest technology.

2) A collection of information and list of devices to be updated: With the collected data, a document is produced with a list of the most common devices in a dental office, organized from most to least used to facilitate the selection of the necessary equipment.

3) Application of Technology Roadmaps for the search: In the SoftProsp platform, we proceed to use the Technological Maps technique to consult dental devices or technologies, writing the words that make up the name of each device, we look for each one to have the information necessary.

In this phase, Bibliometric Analysis is applied to extract metadata from articles, academic publications, citations and patents based on scientific data. The technique groups the information so that the Technology Roadmaps Performs its work of analysis of the technologies.

When classifying the information, Technology Roadmaps performs a semantic analysis to obtain devices and technologies that are related to the established search, visualizing the devices and technologies in chronological order.

4) Selection of technologies and / or devices: At the end of your search you get the technologies and the names of the documents found, the export proceeds to organize and classify technologies as devices in chronological order. The objective is to know the different selection options for the replacement of dental devices in the office or laboratory.

\section{III.RESULTS}

The research was developed with teachers from the dentistry faculty of the University of Cartagena. Next, the results are exposed through the proposed model mentioned above:

\section{1) Preparation and conduct of surveys of expert population}

Preparation and conduct of surveys to population of experts: In the first phase the questions for the survey were prepared, which were validated with the help of five expert dentists of the dental school, and applied to the study participants to establish the dental technology devices common in an office (Fig. 3).

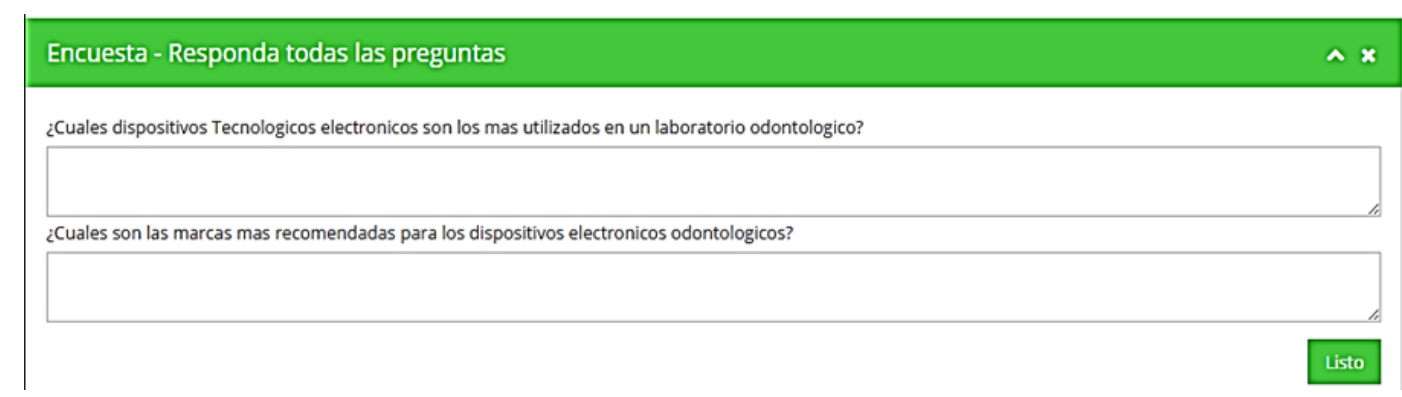

Fig. 3: Outline of survey to dentists.

\section{2) A collection of information and list of devices to be updated}

The information on the survey made by the dental experts was obtained to create a list of devices more named by the participants to facilitate the search in the Technological Maps technique, the list of these items is shown in Table 1: 
TABLE I. List of devices used in the endodontic laboratory

\begin{tabular}{|l|l|l|}
\hline Device & Brand & Reference \\
\hline Dental apex locator & Woodpecker & Woodpex I \\
\hline Autoclave or sterilizer & Ritter and Mindmark & $\begin{array}{l}\text { Mindmark M9 II Automatic } \\
\text { Sterilizer Ritter Cleantec AB 80 }\end{array}$ \\
\hline Photocurate Lamps & Dentsply & Spectrum 800 \\
\hline Cavitron ultrasonic scaler & BonArt & ART-M1 \\
\hline $\begin{array}{l}\text { Portable Rx: intraoral digital } \\
\text { sensor radiovisiograph }\end{array}$ & DigiMed & DVS-100 \\
\hline
\end{tabular}

\section{3) Application of Technology Roadmaps for the search}

The list of the survey is consulted in the Technology Roadmaps technique and to know the current trends in the market, in Figure 4 it is shown the introduction of data of a dental apex for the search of devices between the year 2013 and 2016:

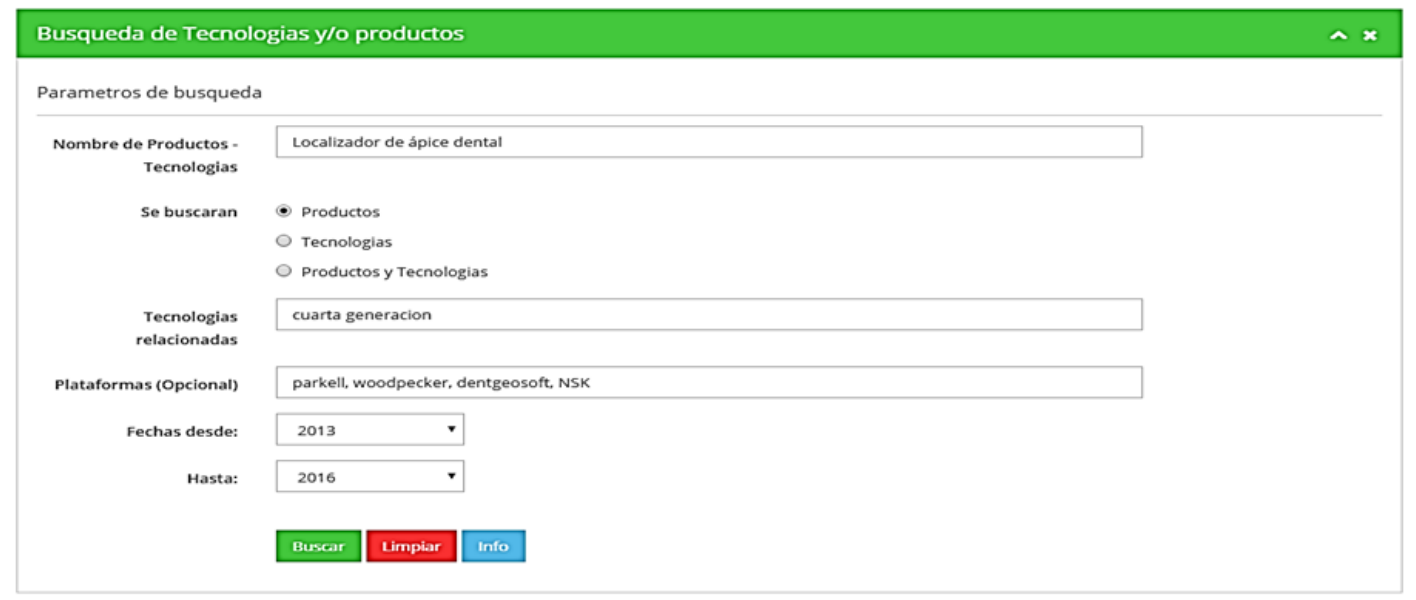

Fig. 4: Introduction of Parameters for searching.

When finished entering the data, the tool shows two tables with the Technology Roadmap of the device, divided into product planning that is the history of the product models and the evolution of the product are the specific characteristics of each of these.

When the search is carried out, the bibliometric analysis technique is responsible for extracting the metadata from the scientific articles related to dental devices, with the objective that the Technology Roadmaps technique can chronologically organize the technologies and devices found in the search.

Figure 5 shows the product planning of a dental apex where are the different models that are available with their date of release to the market, their reference and technology, which comes through the years obtaining the maps of the technologies that have been launched over the years with their provenance and precedence written alphanumerically.

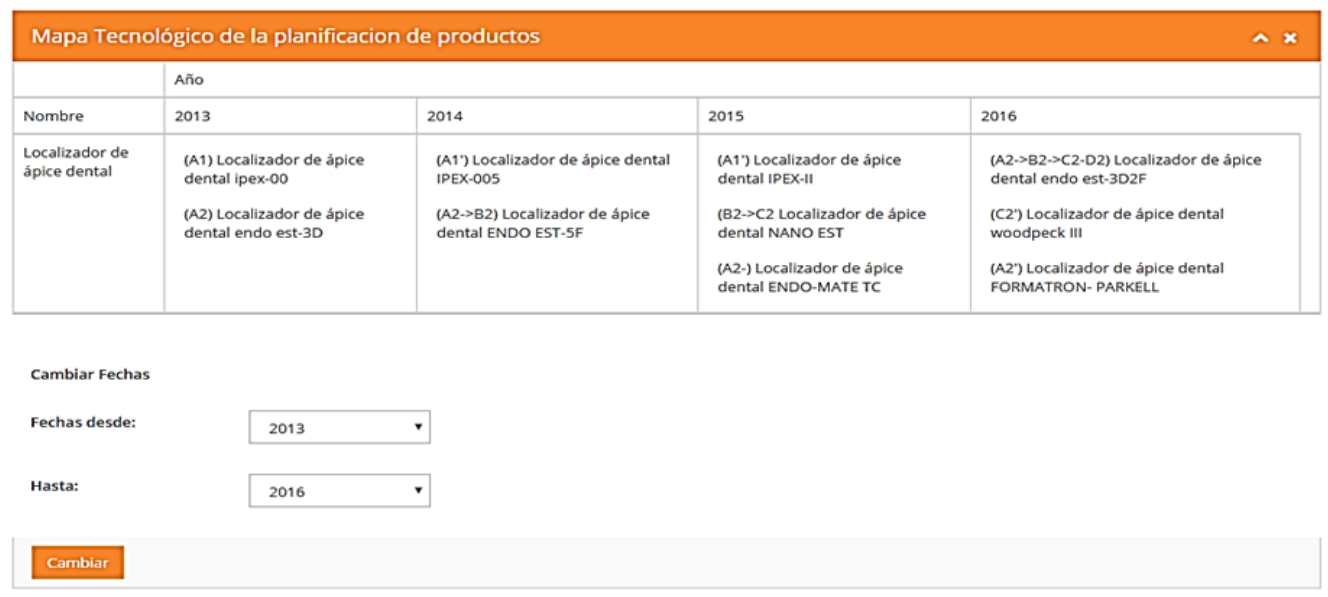

Fig. 5: Technology roadmap of Product planning 
Below the product planning table, you can find the evolution of the product, which shows the characteristics of the product and the need it covers in the market by having a new version, expanding the amount of detail that the tool brings to the technologies present in the query, shown in Figure 6.

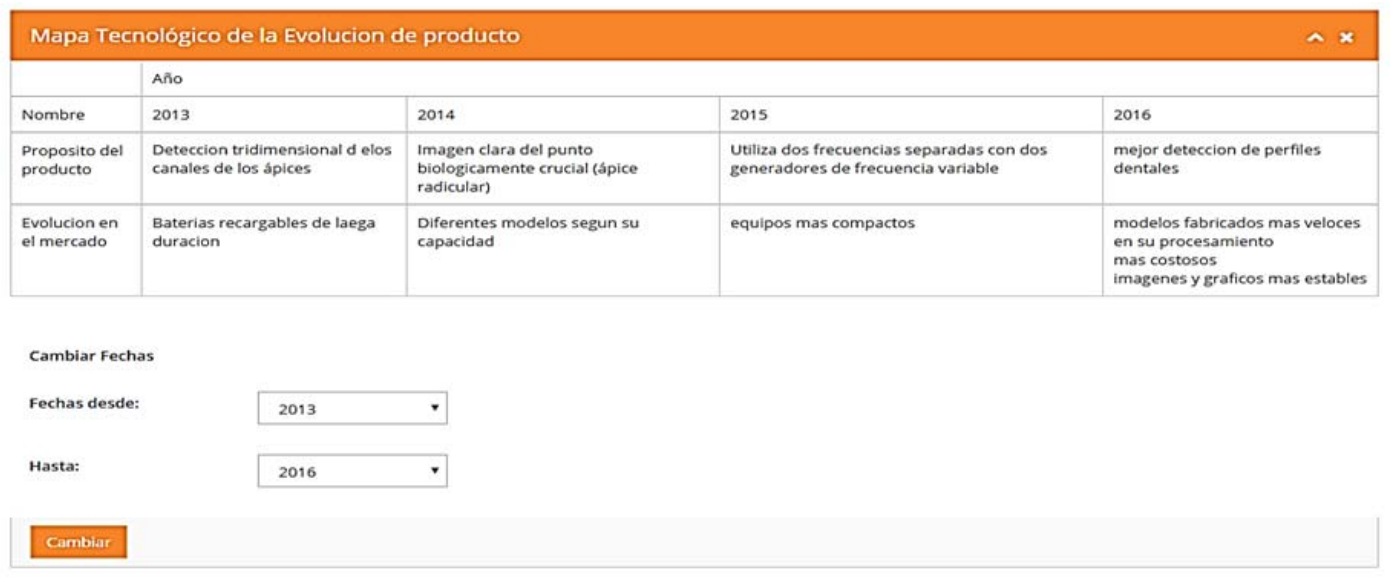

Fig. 6: Technology roadmap of product evolution

\section{4) Selection of technologies and / or devices}

The dental devices are selected according to the results obtained. To replace them in the office, the resources were analyzed, such as the current economic capacity and infrastructure, the availability of the devices in the national market and thus making a decision as to which is appropriate for the entity.

The set of prospective techniques used in this study aims to provide current advances about dental devices in general, which allows recognizing products in the market, exploring better technologies to replace or improve traditional processes that are currently used [18] and provide a space among the experts for the analysis of which are the adequate equipment for updating or obtaining in dental facilities.

\section{IV.CONCLUSION}

Dentistry offices benefit from using these prospective tools to update their technological laboratory devices, eliminating the traditional way of reviewing the state of the devices through the request of an expert in dental technologies, through the hiring of a provider or a public collector. The previous request causes disadvantages to the office due to the increase in costs, in addition to the devices to be replaced, the entity will not have the possibility of selecting any and may even be all of the same brand if the provider is from a specialized company, leaving it without alternatives of choice.

From the results obtained, the following conclusions can be announced: 1) The application of these three prospective techniques helps research in the preparation of documents on specific topics for the provision of devices in a dental office; 2) eliminates the need for a provider or expert seer of a public entity on the subject of dental technology devices for updating clinics; 3) simplifies the research processes of new technologies; 4) queries about technologies are displayed in chronological order according to their launch to know the market trends.

\section{ACKNOWLEDGMENT}

The heading of the Acknowledgment section and the References section must not be numbered.

Causal Productions wishes to acknowledge Michael Shell and other contributors for developing and maintaining the IEEE LaTeX style files which have been used in the preparation of this template. To see the list of contributors, please refer to the top of file IEEETran.cls in the IEEE LaTeX distribution.

\section{REFERENCES}

[1] L.T. Bermúdez and L.F. Rodríguez, Research in business management, 1st ed., p. 115. Bogotá: Ecoe Editions, 2013.

[2] Y. Namihas, "Application of dental instrumental technology in the development of endodontic learning in dental students of the University of San Martin de Porres,” M. thesis, University of San Martin de Porres, Perú, 2014.

[3] Resolution $N^{\circ}$ 2003, Definition of the procedures and conditions thath must be met by tre Healt Service Providers to enable service, Ministry of Healt and Social Protection, 2014.

[4] Y. Geum and Y. Park, "The state-of-the-art of public-sector technology roadmaps: A taxonomical approach to energy technology roadmaps,” Science \& Public Policy, pp. 327-339, 2013.

[5] C. McClees and C. Willyard, “Motorola’s technology roadmaps process,” Res. Manag., vol. 30, issue 5, pp. 13-19, 1987.

[6] P. Letaba, M. Pretorius and L. Pretorius, "Analysis of the intellectual structure and evolution of technology roadmapping literature," Proceedings of PICMET '15: Management of the technology age, pp. 2248-2254, 2015.

[7] C. Haddad and M. Uriona, “A function approach to improve sectoral technology roadmaps,” Technological Forecasting and Social Change, vol. 115, pp. 251-260, 2017.

[8] A. Espinel, V. Martin and A. Vega, "Design of a system for monitoring technology multiple application in order to measure the Gap in technology companies ‘MiPymes’,” International Journal of Artificial Intelligence and Interactive Multimedia, pp. 54-60, 2011. 
[9] W. McDowall, "Technology roadmaps for transition management: the case of hydrogen energy,” Technological Forecasting and Social Change, vol. 79, issue 3, pp. 520-542, 2012.

[10] X. Fan, Y. Gao, B. Ma and Z. Xia, "Chinese academic contribution to burns: a comprehensive bibliometrics analysis from 1985 to 2014,” Burns, vol. 42, issue 7, pp. 1463-1470, 2016.

[11] K.C. Garc and S. Kumar, "Bibliometrics of global Ebola virus disease research as seen through science citation index expanded during 1987-2015”, Travel Medicine and Infectious Disease, vol. 16, issue 2017, pp. 64-65, 2017

[12] H. Chan, W. Jiang, X. Man, Y. Yang and Y. Yang, "State of the art on food waste research: a bibliometrics study from 1997 to 2004," Journal of Cleaner Production, vol. 140, issue 2, pp. 840-846, 2017.

[13] R. Phaal, "Public-domain Roadmaps,” Centre for technology management, Univ. of Cambridge, pp. 1-35, 2011.

[14] S. Deshpande, S. Dixit and J. Mete, “Application if digital technology in prosthodontics - a review of literature," JIDA: Journal of Indian Dental Association, vol. 10, issue 6, pp. 10-17, 2016.

[15] E. Babbie, The basics of social research, 5th ed. Belmont: Wadsworth Cengage Learning, 2011.

[16] R. Kumar, Research methodology, a step by step guide for beginners, 3rd ed. SAGE, 2003.

[17] P. Robles and M. Rojas, Validation by expert judgement: two qualitative researches in applied linguistics, pp. 1-7. Nebrija.

[18] R.J. Martelo, L. Moncaris and L. Velez, "Integration of the Régnier Abacus, Surveys and Brainstorming in the Definition of Key Variables in Prospective Studies,” Información tecnológica, vol. 27, issue 5, pp. 243-250, 2016.

\section{AUTHOR PROFILE}

Raul J. Martelo works as full-time professor at the University of Cartagena (Colombia). Mr. Martelo completed his magister from Industrial University of Santander (Colombia). Mr. Martelo completed his undergraduate in Systems Engineering at the Industrial University of Santander.

Piedad M. Martelo works as part-time professor at the University of Cartagena (Colombia). Mrs. Martelo completed his postgraduate studies from University of Cartagena (Colombia). Mrs. Martelo completed his undergraduate in Dentistry at the University of Cartagena.

Renzo J. Barrios completed his undergraduate in Systems Engineering at the University of Cartagena (Colombia). 